\title{
A photogrammetric and cephalometric evaluation of facial symmetry and smile in relation to attractiveness.
}

\begin{abstract}
Introduction: Human find symmetrical face more attractive than are asymmetrical faces. The smile is one of the most important facial expressions and is essential in expressing friendliness, agreement, and appreciation. The aim of the present study was to evaluate the relationship between smile and symmetry in attributing to attractiveness.

Materials \& Method: The study was conducted in the Department Of Orthodontics and Dentofacial Orthopedics, on 150 samples.150 good quality radiographs and photographs of the patients were obtained. The subjects were divided into three groups:Group I - Horizontal growth pattern Group II -Average growth pattern Group III-Vertical growth pattern. The frontal facial photograph and PA cephalometric radiograph were used to assess the facial symmetry. Posed smile photograph and Lateral cephalometric radiograph were used to assess the smile. Digimizer Image Analyzer (bvba software) were used for the analysis. The ratings were given by the expert panellist based on attractiveness

Results:In the present study, Left facial symmetry parameters is marginally higher than right side in cephalometric analysis and converse for photographic analysis, right facial symmetry parameters is marginally higher than left side but this is not statistically significant. Thereare no statistically significant difference among the groups for smile -photographic parameters and lateral cephalometric smile parameters. There is statistically significant difference among the groups for Visual Analog Scale readings for attractiveness given by orthodontist, general dentist and layperson for frontal profile for the subjects of three study groups.

Conclusion: The study revealed that in cephalometric analysis, left hemiface is wider than right hemiface while in photographic analysis, right hemiface is wider than left hemiface. Vertical grower shows maximum upper incisor exposure and upper and lower vermilion lip thickness. On the contrary full smile length was minimum in vertical grower. The most favored profile by VAS was horizontal growth pattern.
\end{abstract}

Key Words: Symmetry, Smile, Attractiveness

\section{Introduction:}

Human find symmetrical face more attractive than are asymmetrical faces. Evolutionary psychologist claims that our symmetry can be explained in contest of mate choice because symmetry is an honest indicator of genetic quality of potential mates.[1]Psychologist have long been interested in the cognitive mechanisms and adaptive significance of facial attractiveness.[2]

The smile is one of the most important facial expressions and is essential in expressing friendliness, agreement, and appreciation. An attractive or pleasing smile clearly enhances the acceptance of an individual in the society by improving the initial impression in interpersonal relationships. [3]

\begin{tabular}{|l|l|}
\hline \multicolumn{2}{|c|}{ Access this article online } \\
\hline \multirow{2}{*}{$\begin{array}{l}\text { Website: } \\
\text { www.ujds.in }\end{array}$} & Quick Response Code \\
\hline $\begin{array}{l}\text { DOI: } \\
\text { https://doi.org/10.21276/ujds.2020.6.3.1 }\end{array}$ & \\
\hline
\end{tabular}

The purpose of this study was to assess facial symmetry, smileandfacial esthetics in different facial growth patternsand correlate them with underlying hard tissue structure.

\author{
${ }^{1}$ AKSHITA, ${ }^{2}$ CHAUHAN, A. K., ${ }^{3}$ CHAWLA, R. \\ ${ }^{1}$ Shree Bankey Bihari Dental College, Ghaziabad \\ ${ }^{2}$ Kothiwal Dental College and Research Centre, \\ Moradabad. \\ ${ }^{3}$ Dept. of Orthodontics, International Faculty of Dental \\ Medicine of Rabat, Morocco \\ Address for Corresponding : Dr. Riddhi Chawla \\ Department of Orthodontics, International Faculty of \\ Dental Medicine of Rabat, Morocco \\ Email : riddhichawla@yahoo.co.in
}

Received : 18 August 2020, Published : 31 Dec. 2020

How to cite this article: Akshita, Chauhan, A. K., \& Chawla, R. (2021). A photogrammetric and cephalometric evaluation of facial symmetry and smile in relation to attractiveness. UNIVERSITY JOURNAL OF DENTAL SCIENCES, 6(3). https://doi.org/10.21276/ujds.2020.6.3.1: 01-8 


\section{Method And Material:}

The 150 sample were selected from the students of a dental college. Good quality radiographs (lateral and posterioranterior radiographs) and photographs (frontal and posed smile) of the patients were obtained using a digital camera based on following criteria:

Inclusion criteria: Subjects were in the age group of 1825years, there should be no missing except 3rd molars or malformed teeth causing a tooth size discrepancy, subjects should have a clinically acceptable smile and a good profile and Class-I molar relation.

Exclusion criteria: Subjects with congenital anomaly or craniofacial defect, clinically evident skeletal asymmetry or cross bite.

The subjects were divided into three groupsbased on SNMP(Table 1).

Table 1 Various Groups 14

\begin{tabular}{|c|c|c|}
\hline $\begin{array}{c}\text { Group I- Horizontal growth } \\
\text { pattern }\end{array}$ & $\begin{array}{c}\text { Group II - Average growth } \\
\text { pattern }\end{array}$ & $\begin{array}{c}\text { Group III - Vertical growth } \\
\text { pattern }\end{array}$ \\
\hline $25^{\circ}-29^{\circ}$ & $30^{\circ}-34^{\circ}$ & $35^{\circ}-39^{\circ}$ \\
\hline
\end{tabular}

All photographs were taken under same lightening conditions, magnification and at a fixed distance.

\section{Symmetry Analysis 1}

The frontal facial photograph were used to assess the facial symmetry using following attribute(Figure 1):

P1- right outer canthus, $\mathrm{P} 2$ - left outer canthus, P3- right inner canthus, P4- left outer canthus, P5- right zygomatic arch, P6left zygomatic arch, P7- right alar process, P8- left alar process, P9- right chelion, P10- left chelion, P11- right gonion, P12- left gonion, P13- menton, P14- subnasion.

Mid sagittal plane (MSP) was formed by joining P13 and P14 and was used as a reference line for assessing facial symmetry. The distance of various left and right-side points were measured from MSP using Digimizer Image Analyzer software.

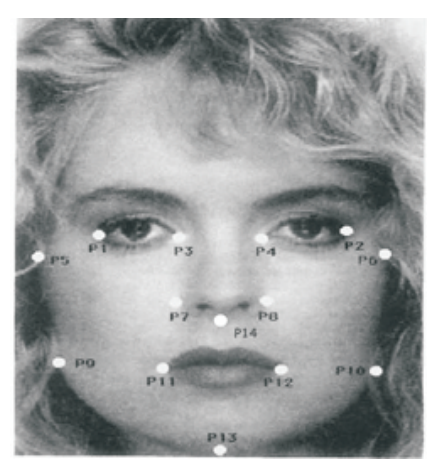

Figure 1: Points used in study Postero-anterior Cephalometric Parameters4

Linear Asymmetry 4-Z-MSP, Co-MSP, J-MSP, Ag-MSP, Me-MSP

Mandibular Asymmetry 4- $\mathrm{Co}-\mathrm{Ag}, \mathrm{Ag}-\mathrm{Me}$, $\mathrm{Co}-\mathrm{Me}$

\section{Smile Analysis 5}

Digimizer Image Analyzer (bvba software) were used to analyze the smile. The following attributes of the smile were measured in millimeters:Max Incisor Exposure, Upper vermillion Lip Thickness, Lower Vermillion Lip Thickness, Full Smile Length.

For the correlation of the cephalometric analysis with the smile analysis, following measurements were used:

\section{Angles5}

SNA, SNB, U1-SN, SN-MP, SN-PP, L1-MP

\section{Linear Measurement5}

Pt A- N perp, Pog - N perp, U1 - Pt A (Horizontal), U1 -Pt A (Vertical), U1-PP(Vertical), N-Me, ANS-Me

Attractiveness were assessed on the basis of visual analogue scale.The ratings were given by the expert panelist based on attractiveness. The rating were given from 1-5 from very unattractive to very attractive.

\section{Statistical analysis:}

Linear and area measurements were compared by two factor analysis of variance (ANOVA) and the significance of mean difference within (intra) and between (inter) the groups was done by Tukey's post hoc test.A two-tailed $\mathrm{p}<0.05$ was considered statistically significant. Analyses were performed on SPSS software (Window version 17.0). 
University J Dent Scie 2020; Vol. 6, Issue 3

Results:

Table 2 Group comparison of Smile parameters using ANOVA

\begin{tabular}{|c|c|c|c|c|c|c|}
\hline & & $\begin{array}{l}\text { Sum } \\
\text { Squares }\end{array}$ & Df & Mean Square & $\mathrm{F}$ & Sig. \\
\hline & Between Groups & 25.653 & 2 & 12.827 & .577 & .563 \\
\hline SNA & Within Groups & 3265.740 & 147 & 22.216 & & \\
\hline & Total & 3291.393 & 149 & & & \\
\hline & Between Groups & 23.790 & 2 & 11.895 & .609 & .545 \\
\hline SNB & Within Groups & 2873.210 & 147 & 19.546 & & \\
\hline & Total & 2897.000 & 149 & & & \\
\hline & Between Groups & 196.163 & 2 & 98.082 & 2.080 & .129 \\
\hline UI- SN & Within Groups & 6930.310 & 147 & 47.145 & & \\
\hline & Total & 7126.473 & 149 & & & \\
\hline & Between Groups & 5.320 & 2 & 2.660 & .356 & .701 \\
\hline PP-SN & Within Groups & 1098.340 & 147 & 7.472 & & \\
\hline & Total & 1103.660 & 149 & & & \\
\hline & Between Groups & 49.053 & 2 & 24.527 & .407 & .666 \\
\hline LI- MP & Within Groups & 8854.520 & 147 & 60.235 & & \\
\hline & Total & 8903.573 & 149 & & & \\
\hline & Between Groups & .303 & 2 & .152 & .064 & .938 \\
\hline Pt A- N perp & Within Groups & 350.070 & 147 & 2.381 & & \\
\hline & Total & 350.373 & 149 & & & \\
\hline & Between Groups & 6.943 & 2 & 3.472 & .283 & .754 \\
\hline Pog $-\mathrm{N}$ & Within Groups & 1802.350 & 147 & 12.261 & & \\
\hline & Total & 1809.293 & 149 & & & \\
\hline & Between Groups & 7.053 & 2 & 3.527 & .707 & .495 \\
\hline UI- Pt A & Within Groups & 733.240 & 147 & 4.988 & & \\
\hline & Total & 740.293 & 149 & & & \\
\hline & Between Groups & 37.889 & 2 & 18.944 & .922 & .400 \\
\hline Ul- Pt A & Within Groups & 3020.283 & 147 & 20.546 & & \\
\hline & Total & 3058.172 & 149 & & & \\
\hline & Between Groups & 80.253 & 2 & 40.127 & .795 & .453 \\
\hline $\mathrm{N}-\mathrm{Me}$ & Within Groups & 7416.420 & 147 & 50.452 & & \\
\hline & Total & 7496.673 & 149 & & & \\
\hline & Between Groups & 126.093 & 2 & 63.047 & 1.613 & .203 \\
\hline ANS - Me & Within Groups & 5745.240 & 147 & 39.083 & & \\
\hline & Total & 5871.333 & 149 & & & \\
\hline
\end{tabular}

Table 2 shows inter group comparison of lateral cephalometric smile parameters using ANOVA. It shows that there is no statistically significant difference among the groups 
University J Dent Scie 2020; Vol. 6, Issue 3

Table 3Group comparison of facial symmetry parameters using ANOVA

\begin{tabular}{|c|c|c|c|c|c|c|}
\hline & & Sum of Squares & Df & Mean Square & $\mathrm{F}$ & Sig. \\
\hline \multirow{3}{*}{ Rt Z- MSP } & Between Groups & 20.070 & 2 & 10.035 & .944 & .391 \\
\hline & Within Groups & 1562.690 & 147 & 10.631 & & \\
\hline & Total & 1582.760 & 149 & & & \\
\hline \multirow{3}{*}{ 1t Z- MSP } & Between Groups & 12.653 & 2 & 6.327 & .494 & .611 \\
\hline & Within Groups & 1884.180 & 147 & 12.818 & & \\
\hline & Total & 1896.833 & 149 & & & \\
\hline \multirow{3}{*}{ Rt Co-MSP } & Between Groups & 39.960 & 2 & 19.980 & 1.299 & .276 \\
\hline & Within Groups & 2260.680 & 147 & 15.379 & & \\
\hline & Total & 2300.640 & 149 & & & \\
\hline \multirow{3}{*}{ 1t Co-MSP } & Between Groups & 7.960 & 2 & 3.980 & .287 & .751 \\
\hline & Within Groups & 2038.200 & 147 & 13.865 & & \\
\hline & Total & 2046.160 & 149 & & & \\
\hline \multirow{3}{*}{ rt $J-M S P$} & Between Groups & .373 & 2 & .187 & .039 & .962 \\
\hline & Within Groups & 701.200 & 147 & 4.770 & & \\
\hline & Total & 701.573 & 149 & & & \\
\hline \multirow{3}{*}{ 1t $\mathrm{J}-\mathrm{MSP}$} & Between Groups & 2.080 & 2 & 1.040 & .136 & .873 \\
\hline & Within Groups & 1125.980 & 147 & 7.660 & & \\
\hline & Total & 1128.060 & 149 & & & \\
\hline \multirow{3}{*}{$\mathrm{rt} \mathrm{Ag}-\mathrm{MSP}$} & Between Groups & 23.413 & 2 & 11.707 & .500 & .608 \\
\hline & Within Groups & 3442.060 & 147 & 23.415 & & \\
\hline & Total & 3465.473 & 149 & & & \\
\hline \multirow{3}{*}{ lt Ag - MSP } & Between Groups & 12.653 & 2 & 6.327 & .305 & .738 \\
\hline & Within Groups & 3053.320 & 147 & 20.771 & & \\
\hline & Total & 3065.973 & 149 & & & \\
\hline \multirow{3}{*}{$\mathrm{rt} \mathrm{Me}-\mathrm{MSP}$} & Between Groups & .213 & 2 & .107 & .153 & .858 \\
\hline & Within Groups & 102.160 & 147 & .695 & & \\
\hline & Total & 102.373 & 149 & & & \\
\hline \multirow{3}{*}{ 1t $\mathrm{Me}-\mathrm{MSP}$} & Between Groups & .173 & 2 & .087 & .120 & .887 \\
\hline & Within Groups & 106.420 & 147 & .724 & & \\
\hline & Total & 106.593 & 149 & & & \\
\hline \multirow{3}{*}{ rt Co-Ag } & Between Groups & 22.573 & 2 & 11.287 & .158 & .854 \\
\hline & Within Groups & 10533.300 & 147 & 71.655 & & \\
\hline & Total & 10555.873 & 149 & & & \\
\hline \multirow{3}{*}{ Lt Co-Ag } & Between Groups & 17.440 & 2 & 8.720 & .131 & .877 \\
\hline & Within Groups & 9776.700 & 147 & 66.508 & & \\
\hline & Total & 9794.140 & 149 & & & \\
\hline \multirow{3}{*}{ Rt Ag $-\mathrm{Me}$} & Between Groups & 18.013 & 2 & 9.007 & .543 & .582 \\
\hline & Within Groups & 2440.280 & 147 & 16.601 & & \\
\hline & Total & 2458.293 & 149 & & & \\
\hline \multirow{3}{*}{ It $\mathrm{Ag}-\mathrm{Me}$} & Between Groups & 41.293 & 2 & 20.647 & .640 & .529 \\
\hline & Within Groups & 4745.300 & 147 & 32.281 & & \\
\hline & Total & 4786.593 & 149 & & & \\
\hline \multirow{3}{*}{$\mathrm{Rt} \mathrm{Co}-\mathrm{Me}$} & Between Groups & 35.453 & 2 & 17.727 & .306 & .737 \\
\hline & Within Groups & 8525.320 & 147 & 57.995 & & \\
\hline & Total & 8560.773 & 149 & & & \\
\hline \multirow{3}{*}{$\mathrm{Lt} \mathrm{Co}-\mathrm{Me}$} & Between Groups & 28.093 & 2 & 14.047 & .223 & .800 \\
\hline & Within Groups & 9254.900 & 147 & 62.959 & & \\
\hline & Total & 9282.993 & 149 & & & \\
\hline
\end{tabular}

Table 3 shows inter group comparison of facial symmetry parameters using ANOVA. It shows that there is no statistically significant difference among the groups 
University J Dent Scie 2020; Vol. 6, Issue 3

Table 4 Group comparison for linear measurement facial symmetry photograph using ANOVA

\begin{tabular}{|c|c|c|c|c|c|c|}
\hline & & Sum of Squares & $\mathrm{Df}$ & Mean Square & $\mathrm{F}$ & Sig. \\
\hline \multirow{3}{*}{ P1 - MSP } & Between Groups & 36.275 & 2 & 18.137 & 1.511 & .224 \\
\hline & Within Groups & 1764.624 & 147 & 12.004 & & \\
\hline & Total & 1800.898 & 149 & & & \\
\hline \multirow{3}{*}{$\mathrm{P} 2-\mathrm{MSP}$} & Between Groups & 29.035 & 2 & 14.517 & 1.016 & .365 \\
\hline & Within Groups & 2100.755 & 147 & 14.291 & & \\
\hline & Total & 2129.790 & 149 & & & \\
\hline \multirow{3}{*}{ P3 - MSP } & Between Groups & 13.266 & 2 & 6.633 & 2.443 & .090 \\
\hline & Within Groups & 399.113 & 147 & 2.715 & & \\
\hline & Total & 412.379 & 149 & & & \\
\hline \multirow{3}{*}{ P4 - MSP } & Between Groups & 7.143 & 2 & 3.571 & 1.177 & .311 \\
\hline & Within Groups & 446.112 & 147 & 3.035 & & \\
\hline & Total & 453.255 & 149 & & & \\
\hline \multirow{3}{*}{ P5 - MSP } & Between Groups & 11.702 & 2 & 5.851 & .268 & .765 \\
\hline & Within Groups & 3210.921 & 147 & 21.843 & & \\
\hline & Total & 3222.623 & 149 & & & \\
\hline \multirow{3}{*}{ P6 - MSP } & Between Groups & 15.520 & 2 & 7.760 & .329 & .720 \\
\hline & Within Groups & 3471.422 & 147 & 23.615 & & \\
\hline & Total & 3486.942 & 149 & & & \\
\hline \multirow{3}{*}{ P7 - MSP } & Between Groups & .758 & 2 & .379 & .111 & .895 \\
\hline & Within Groups & 502.968 & 147 & 3.422 & & \\
\hline & Total & 503.726 & 149 & & & \\
\hline \multirow{3}{*}{ P8 - MSP } & Between Groups & 3.777 & 2 & 1.888 & .624 & .537 \\
\hline & Within Groups & 444.874 & 147 & 3.026 & & \\
\hline & Total & 448.651 & 149 & & & \\
\hline \multirow{3}{*}{ P9 - MSP } & Between Groups & 40.102 & 2 & 20.051 & .500 & .608 \\
\hline & Within Groups & 5898.145 & 147 & 40.123 & & \\
\hline & Total & 5938.247 & 149 & & & \\
\hline \multirow{3}{*}{ P10 - MSP } & Between Groups & 66.656 & 2 & 33.328 & .795 & .454 \\
\hline & Within Groups & 6163.479 & 147 & 41.928 & & \\
\hline & Total & 6230.135 & 149 & & & \\
\hline \multirow{3}{*}{ P11 - MSP } & Between Groups & 1.796 & 2 & .898 & .168 & .845 \\
\hline & Within Groups & 785.155 & 147 & 5.341 & & \\
\hline & Total & 786.950 & 149 & & & \\
\hline \multirow{3}{*}{ P12 - MSP } & Between Groups & .003 & 2 & .001 & .000 & 1.000 \\
\hline & Within Groups & 727.371 & 147 & 4.948 & & \\
\hline & Total & 727.374 & 149 & & & \\
\hline
\end{tabular}

Table4 describes the inter group comparison for linear measurement facial symmetry- photographs parameters using ANOVA. It shows that there is no statistically significant difference among the groups 
University J Dent Scie 2020; Vol. 6, Issue 3

Table5 Group comparison for smile parameters photographic using ANOVA

\begin{tabular}{|c|c|c|c|c|c|c|}
\hline & & Sum of Squares & Df & Mean Square & $\mathrm{F}$ & Sig. \\
\hline \multirow{3}{*}{$\begin{array}{l}\text { Maximum } \\
\text { Exposure }\end{array}$} & Between Groups & 5.274 & 2 & 2.637 & 1.438 & .241 \\
\hline & Within Groups & 269.546 & 147 & 1.834 & & \\
\hline & Total & 274.820 & 149 & & & \\
\hline \multirow{3}{*}{$\begin{array}{l}\text { Upper Vermillion Lip } \\
\text { Thickness }\end{array}$} & Between Groups & 3.218 & 2 & 1.609 & .625 & .537 \\
\hline & Within Groups & 378.446 & 147 & 2.574 & & \\
\hline & Total & 381.664 & 149 & & & \\
\hline \multirow{3}{*}{$\begin{array}{l}\text { Lower Vermillion Lip } \\
\text { Thickness }\end{array}$} & Between Groups & 4.158 & 2 & 2.079 & .624 & .537 \\
\hline & Within Groups & 489.899 & 147 & 3.333 & & \\
\hline & Total & 494.056 & 149 & & & \\
\hline \multirow{3}{*}{ Full Smile Length } & Between Groups & 61.755 & 2 & 30.878 & .855 & .427 \\
\hline & Within Groups & 5307.870 & 147 & 36.108 & & \\
\hline & Total & 5369.625 & 149 & & & \\
\hline
\end{tabular}

Table 5 describes inter group comparison for smile -photographic parameters using ANOVA

It shows that there is no statistically significant difference among the groups.

Table 6 Group comparison for VAS scores using ANOVA

\begin{tabular}{|c|c|c|c|c|c|c|c|}
\hline & & & $\begin{array}{l}\text { Sum of } \\
\text { Squares }\end{array}$ & Df & $\begin{array}{l}\text { Mean } \\
\text { Square }\end{array}$ & $\mathrm{F}$ & Sig. \\
\hline \multirow{6}{*}{ ORTHODONTIST } & \multirow{3}{*}{ FRONTAL } & Between Groups & 1.973 & 2 & .987 & 2.313 & .103 \\
\hline & & Within Groups & 62.720 & 147 & .427 & & \\
\hline & & Total & 64.693 & 149 & & & \\
\hline & \multirow{3}{*}{ SMILING } & Between Groups & 1.480 & 2 & .740 & 1.701 & .186 \\
\hline & & Within Groups & 63.960 & 147 & .435 & & \\
\hline & & Total & 65.440 & 149 & & & \\
\hline \multirow{6}{*}{$\begin{array}{l}\text { GENERAL } \\
\text { DENTIST }\end{array}$} & \multirow{3}{*}{ FRONTAL } & Between Groups & 1.480 & 2 & .740 & 1.701 & .186 \\
\hline & & Within Groups & 63.960 & 147 & .435 & & \\
\hline & & Total & 65.440 & 149 & & & \\
\hline & \multirow{3}{*}{ SMILING } & Between Groups & 1.973 & 2 & .987 & 2.313 & .103 \\
\hline & & Within Groups & 62.720 & 147 & .427 & & \\
\hline & & Total & 64.693 & 149 & & & \\
\hline \multirow{6}{*}{ LAYPERSON } & \multirow{3}{*}{ FRONTAL } & Between Groups & 1.973 & 2 & .987 & 2.313 & .103 \\
\hline & & Within Groups & 62.720 & 147 & .427 & & \\
\hline & & Total & 64.693 & 149 & & & \\
\hline & \multirow{3}{*}{ SMILING } & Between Groups & 1.480 & 2 & .740 & 1.701 & .186 \\
\hline & & Within Groups & 63.960 & 147 & .435 & & \\
\hline & & Total & 65.440 & 149 & & & \\
\hline
\end{tabular}

Table 6 shows group comparison for VAS readings for attractiveness given by orthodontist, general dentist and layperson for frontal and smiling profile using ANOVA. It showsthat there is statistically significant difference among the groups. 


\section{Discussion:}

Perfectly bilateral face and body is largely a theoretical concept that seldom exists. Right-left differences occur everywhere in nature where two bilateral congruent parts presents in an entity.[6]

Smile analysis and design have become key elements of orthodontic diagnosis and treatment planning.In the evolution of orthodontics, the changes in the soft tissue attract a considerable attention.[7]

All images have some degree of asymmetry which can be attributed to (a) Each half of our brain has a different function (b) The chance is almost zero that all milliards of cells that build our faces will be distributed in a complete symmetry from our birth to death.[8]

\section{Cephalometric and Photographic Symmetry Parameters:}

All the parameters were slightly higher on left side than on right side except Me - MSP which was higher on right side than on left side.

Most studies of normal asymmetry have reported the reverse relation.[1,9] However, some reports have found the left hemiface to be wider.[10,11] In this study, leftsidewas marginally higher than Rightside incontrary to Simmons et al1 and Haraguchi et al9 whereas above finding is supported by the study done by Vig et al10and Chebib et al.[11]

The study conducted by Farkas et al12shows that most common and large asymmetries were found in upper third of face which is seen in the present study.

\section{Cephalometric and Photographic Smile Parameters:}

Maximum incisor exposure-The incisal display significantly increased from average to horizontal to vertical facial growth pattern. Contrary to this, McNamara et al.13 found that the vertical display on smile of the maxillary right central incisor could not be correlated with the skeletal vertical dimension.

Upper Vermilion lip thickness-Upper Vermillion Lip Thickness is maximum in vertical growth pattern and minimum in horizontal growth pattern.However, Grover et al14showed opposite results.
Lower Vermilion lip thickness -The Lower Vermillion Lip Thickness is maximum in vertical growth pattern and minimum in horizontal growth pattern.

Full smile Length -The Full smile length was maximum in average growth pattern and minimum in vertical growth pattern. The similar results wereobserved by Grover et al14. This was contrary to the results of Rigsbee et al.15 and Chetan et al.[7]

\section{Visual Analog Scale :}

In this study there is high significant difference in the profile and smiling photograph in average growth pattern by layman and orthodontist. The study done by Rai et al5 showed a statistically high significant difference between the perception of the smile by the orthodontist and layperson. The most favored profile by VAS was horizontal growth pattern, followed by vertical growth pattern and average growth pattern. This is similar to finding done by Lundstrom et al16

\section{Conclusion:}

\section{The following conclusion can be drawn:}

1. In cephalometric analysis, left hemiface is wider than right hemiface while in photographic analysis, right hemiface is wider than left hemiface. There was strong correlation seen among skeletal symmetry and soft tissue parameters. Soft tissue camouflaged the underling hard tissue.

2. Vertical grower shows maximum upper incisor exposure and upper and lower vermilion lip thickness. On the contrary full smile length was minimum in vertical grower.

3. The most favored profile by VAS was horizontal growth pattern.

\section{References:}

1. Simmons LW, Rhodes G, Peters M, Koehler N. Are human preferences for facial symmetry focused on signals of developmental instability. Behavioral Ecology 2004; 15:864-871.

2. Perrett DI, Burt DM, Penton-Voak IS, Lee KJ, Rowland DA, Edwards R. Symmetry and human facial attractiveness. Evolution and human behavior 1999;20:295-307.

3. Krishnan V, Daniel ST, Lazar D, Asok A. characterization of posed smile by using visual analog 
scale, smile arc, buccal corridor measures, and modified smile index. Am J Orthod Dentofacial Orthop2008;133:515-23.

4. Grummons DC. A frontal asymmetry analysis. JCO 1987;21:448-465.

5. Rai D, Janardhanam P, Rai A. Esthetic factors of smile in vertical dimensions: A comparative evaluation. J Indian Orthod Society 2015;49:25-31.

6. Cheong YW, Lo LJ. Facial Asymmetry: Etiology, Evaluation, and Management. Chang Gung Med J 2011;34:341-51.

7. Chetan P, Tandon P, Singh GK, Nagar A, Prasad V, Chugh VK. Dynamics of a smile in different age groups. Angle Orthod.2013;83:90-96.

8. Tamir, Abraham MD. Quantitative Analysis of Face Symmetry. J Craniofacial Surgery 2015;26:1268-1269.

9. Haraguchi S, Iguchi Y, Takada K. Asymmetry of the Face in Orthodontic Patients. Angle Orthod 2008; 78: 421-426.

10. Vig PS, Hewitt AB. Asymmetry of the human facial skeleton. Angle Orthod. 1975;45:125-129.

11. Chebib FS, Chamma AM. Indices of craniofacial asymmetry.AngleOrthod. 1981;51:214-226.

12. Farkas LG, Cheung G. Facial asymmetry in healthy North American caucasians. Angle Orthod. 1981; 61:70-77.

13. McNamara L, McNamara Jr JA, Ackerman MB, Baccetti T. Hard- and softtissue contributions to the esthetics of the posed smile in growing patients seeking orthodontic treatment. Am J OrthodDentofacOrthop. 2008;133:491-9.

14. Grover N, Kapoor DN, Verma S, Bharadwaj P. Smile analysis in different facial patterns and its correlation with underlying hard tissues. Prog Orthod. 2015;16:28.

15. Rigsbee OH, Sperry TP, BeGole EA. The influence of facial animation on smile characteristics. Int J Adult OrthodOrthognath Surg. 1988;3:233-9.

16. Lundstrom A, Woodside DG, Popovich F. panel assessment of facial profile related to mandibular growth direction. Eur J Orthod 1987; 9:271-278. 